\title{
Effectiveness of adjuvant systemic chemotherapy for intermediate- risk stage IB cervical cancer
}

\author{
Koji Matsuo ${ }^{1,2, *}$, Muneaki Shimada ${ }^{3,4, *}$, Harushige Yokota5, Toyomi Satoh ${ }^{6}$, Hidetaka \\ Katabuchi', Shoji Kodama ${ }^{8,9}$, Hiroshi Sasaki ${ }^{10,11}$, Noriomi Matsumura ${ }^{12,13}$, Mikio \\ Mikami ${ }^{14}$ and Toru Sugiyama ${ }^{15}$ \\ ${ }^{1}$ Division of Gynecologic Oncology, Department of Obstetrics and Gynecology, University of Southern California, Los Angeles, \\ CA, USA \\ ${ }^{2}$ Norris Comprehensive Cancer Center, University of Southern California, Los Angeles, CA, USA \\ ${ }^{3}$ Department of Obstetrics and Gynecology, Tottori University, Tottori, Japan \\ ${ }^{4}$ Current affiliation: Department of Obstetrics and Gynecology, Tohoku University, Miyagi, Japan \\ ${ }^{5}$ Department of Gynecology, Saitama Cancer Center, Saitama, Japan \\ ${ }^{6}$ Department of Obstetrics and Gynecology, University of Tsukuba Faculty of Medicine, Tsukuba, Japan \\ ${ }^{7}$ Department of Obstetrics and Gynecology, Faculty of Life Sciences Kumamoto University, Kumamoto, Japan \\ ${ }^{8}$ Department of Gynecology, Niigata Cancer Center Hospital, Niigata, Japan \\ ${ }^{9}$ Current affiliation: Department of Obstetrics and Gynecology, Niigata Minami Hospital, Niigata, Japan \\ ${ }^{10}$ Department of Obstetrics and Gynecology, The Jikei University Kashiwa Hospital, Kashiwa, Japan \\ ${ }^{11}$ Current affiliation: Department of Gynecology, Chiba Tokushukai Hospital, Funabashi, Japan \\ ${ }^{12}$ Department of Obstetrics and Gynecology, Kyoto University, Kyoto, Japan \\ ${ }^{13}$ Current affiliation: Department of Obstetrics and Gynecology, Kindai University Faculty of Medicine, Osaka-Sayama, Japan \\ ${ }^{14}$ Department of Obstetrics and Gynecology, Tokai University, Kanagawa, Japan \\ ${ }^{15}$ Department of Obstetrics and Gynecology, Iwate Medical University, Iwate, Japan \\ *These authors contributed equally to this work
}

Correspondence to: Muneaki Shimada, email: muneaki.shimada.b7@tohoku.ac.jp

Koji Matsuo, email: koji.matsuo@med.usc.edu

Keywords: cervical cancer, radical hysterectomy, intermediate risk, adjuvant, chemotherapy

Received: August 10, $2017 \quad$ Accepted: September 29, $2017 \quad$ Published: November 15, 2017

Copyright: Matsuo et al. This is an open-access article distributed under the terms of the Creative Commons Attribution License 3.0 (CC BY 3.0), which permits unrestricted use, distribution, and reproduction in any medium, provided the original author and source are credited.

\section{ABSTRACT}

Objective: To examine the effectiveness of systemic chemotherapy following radical hysterectomy for women with intermediate-risk stage IB cervical cancer.

Materials and Methods: This is a retrospective analysis of a previously organized nation-wide cohort study examining 6,003 women with stage IB-IIB cervical cancer who underwent radical hysterectomy between 2004 and 2008 in Japan. Survival of 555 women with stage IB cervical cancer in the intermediate-risk group (deep stromal invasion $>50 \%$, large tumor size $>4 \mathrm{~cm}$, and lympho-vascular space invasion [LVSI]) were examined based on adjuvant therapy patterns: chemotherapy alone $(n=223,40.2 \%)$, concurrent chemo-radiotherapy $(n=172,31.0 \%)$, and radiotherapy alone $(n=160,28.8 \%)$.

Results: The most common intermediate-risk pattern was LVSI with deep stromal invasion $(n=216,38.5 \%)$. The most common chemotherapeutic choice was taxane/ platinum (52.2\%). Women with adenocarcinoma/adenosquamous histology were more likely to receive chemotherapy $(P=0.03)$, and intermediate-risk pattern was not associated with chemotherapy use $(P=0.11)$. Women who received systemic chemotherapy had disease-free survival (5-year rate, $88.1 \%$ versus $90.2 \%$, adjustedhazard ratio (HR) $0.98,95 \%$ confidence interval $(\mathrm{CI}) 0.52-1.83, P=0.94$ ) and cause- 
specific survival (95.4\% versus $94.8 \%$, adjusted-HR $0.85,95 \%$ CI $0.34-2.07, P=$ 0.71 ) similar to those who received concurrent chemo-radiotherapy on multivariable analysis. Similar results were seen among 329 women with multiple intermediaterisk factors (5-year rates for disease-free survival, chemotherapy versus concurrent chemo-radiotherapy, $87.1 \%$ versus $90.2 \%, P=0.86$; and cause-specific survival 94.6\% versus $93.4 \%, P=0.82)$. Cumulative local-recurrence $(P=0.77)$ and distantrecurrence $(P=0.94)$ risks were similar across the adjuvant therapy types.

Conclusions: Our study suggests that systemic chemotherapy may be an alternative treatment choice for adjuvant therapy in intermediate-risk stage IB cervical cancer.

\section{INTRODUCTION}

Worldwide, cervical cancer was the most common gynecologic malignancy in 2012 [1]. When the gross tumor is confined to the cervix, surgery remains the mainstay of treatment, consisting of radical hysterectomy and pelvic lymphadenectomy [2]. Hysterectomy specimens are useful to identify certain tumor factors associated with an increased risk of disease recurrence. Historically, these surgical-pathological risk factors are grouped into high-risk and intermediate-risk [3-5], and tumor factors in the intermediate-risk group include a large tumor size, deep stromal invasion, and lymphovascular space invasion (LVSI).

Per the current guidelines set forth by multiple societies, women with early-stage cervical cancer in the intermediate-risk group are recommended to receive postoperative pelvic radiotherapy after surgical treatment in order to reduce the risk of recurrence $[6,7]$. While the survival benefit of this adjuvant radiotherapy is supported by level I evidence [4], there is a concern for adverse events related to radiotherapy including genitourinary, hematologic, and gastrointestinal complications [8].

Due to these toxicity profiles of radiotherapy use, systemic chemotherapy has been considered as an alternative treatment option for adjuvant therapy after radical hysterectomy for women in the intermediate-risk group [9-12]. While some studies have suggested the possible utility of systemic chemotherapy, they were conducted with a relatively small sample size or with a lack of an appropriate control arm making their results difficult to interpret.

The objective of this study was to examine survival outcomes of women with intermediate-risk stage IB cervical cancer who received postoperative systemic chemotherapy following radical hysterectomy, and compare these outcomes to those women who received radiation-based therapy.

\section{RESULTS}

The selection schema is shown in Figure 1. Among 6,003 cases in the study cohort, there were 555 cases which met the eligibility criteria for this analysis. Adjuvant therapy patterns included systemic chemotherapy $(n=223)$, concurrent chemo-radiotherapy (CCRT) $(n=172)$, and radiotherapy (RT) alone $(n=160)$.
In the chemotherapy group (Supplementary Table 1), the most common chemotherapy choice was a taxane/ platinum-doublet (52.9\%) followed by irinotecan-based regimens $(19.7 \%)$. The median number of administered chemotherapy cycles was 4 , and $32.3 \%$ received 6 cycles of chemotherapy. The majority of chemotherapy was administered with a 4-week time interval (61.9\%). Chemotherapy delay was seen in $37(16.6 \%)$ cases with hematological toxicity being the most common reason for this delay $(n=25,67.6 \%)$. Treatment delay was more common among women who received $>3$ cycles compared to those who received $\leq 3$ cycles $(23.7 \%$ versus $10.6 \%, P=0.017)$. There were $23(10.3 \%)$ cases with chemotherapy discontinuation in this study population. In the CCRT group (Supplementary Table 2), the most common radio-sensitizer was cisplatin $(n=88,51.2 \%)$ followed by nedaplatin ( $n=54,31.4 \%)$.

Patient demographics across the three groups are shown in Table 1. Patient age at diagnosis was similar across the groups $(P=0.18)$, and the year of diagnosis was not associated with chemotherapy use $(P=0.36)$. Women with adenocarcinoma / adenosquamous histology were more likely to receive chemotherapy compared to other treatment options: $52.9 \%$ for the chemotherapy group, $24.4 \%$ for the CCRT group, and $25.6 \%$ for the RT group $(P<0.001)$. Women who received chemotherapy had a lower frequency of tumor with deep stromal invasion compared to the other modalities $(P=0.02)$. The frequency of large tumors and LVSI were similar across the three groups (both, $P>0.05$ ). Women in the CCRT group had the lowest pelvic lymph node counts among the three groups whereas women in the RT alone had had the lowest para-aortic lymph node counts among the three groups (both, $P<0.05$ ).

The most common intermediate-risk factor was LVSI $(n=427,76.9 \%)$ followed by deep stromal invasion $(n=392,70.6 \%)$ and large tumor size $(n=125,22.5 \%)$. When cases were stratified by the combination of intermediate-risk factors in the whole cohort, tumors with LVSI and deep stromal invasion was the most common pattern $(n=214,38.6 \%)$ followed by LVSI alone $(n=131$, $23.6 \%)$ and deep stromal invasion alone ( $n=85,15.3 \%)$. There were $63(11.4 \%)$ cases that had all three of these intermediate-risk factors.

Survival analysis was performed and the median follow-up time of women without survival events was 
5.5 years. There were 62 recurrences and 30 deaths due to cervical cancer identified in this study. There were no deaths related to complications from adjuvant chemotherapy or radiotherapy in the cohort. On univariable analysis, adjuvant treatment types were not associated with disease-free survival (5-year rates, $88.0 \%$ for chemotherapy group, $90.2 \%$ for CCRT group, and $89.8 \%$ for RT group, $P=0.90$; Figure $2 \mathrm{~A}$ ). When the association of adjuvant therapy and disease-free survival was adjusted for other covariates on multivariable models (Table 2), chemotherapy use and CCRT did not differ in disease-free survival (adjusted-hazard ratio (HR) 0.98, $95 \%$ confidence interval (CI) 0.52 to $1.84, P=0.95$ ). The systemic chemotherapy group had a similar disease-free survival compared to the RT alone group on adjusted models (data not shown).

Similarly, adjuvant treatment types were not associated with cause-specific survival (5-year rates, $95.3 \%$ for chemotherapy group, $94.8 \%$ for CCRT group, and $94.8 \%$ for RT group, $P=0.99$; Figure $2 \mathrm{~B}$ ). After adjusting for other covariates for cause-specific survival in multiple models (Table 3), chemotherapy use was not associated with cause-specific survival compared to CCRT (adjusted-HR $0.85,95 \%$ CI 0.34 to $2.08, P=0.71$ ). Likewise, cause-specific survival was similar between the systemic chemotherapy group and the RT alone group on adjusting models (data not shown).

When survival was compared between CCRT and RT alone groups, women who received CCRT had disease-free survival (adjusted-HR 1.00, 95\% CI 0.53 to $1.90, P=0.99$ ) and cause-specific survival (adjustedHR $0.82,95 \%$ CI 0.34 to $2.01, P=0.67$ ) similar to those who received RT alone. Among women who received adjuvant chemotherapy, the number of administered cycles was not associated with disease-free survival (5-year rate, 3 versus 6 cycles, $89.4 \%$ versus $89.6 \%$, $P=0.84)$ and cause-specific survival $(97.4 \%$ versus $92.9 \%, P=0.38$ ). Chemotherapy treatment delay was significantly associated with decreased disease-free survival $(70.8 \%$ versus $91.9 \%, P=0.001)$.

Among women who developed recurrent disease (median follow-up time after recurrence 42.6 months for censored cases and 15.3 months for deceased cases), the second remission rates after salvage intervention were

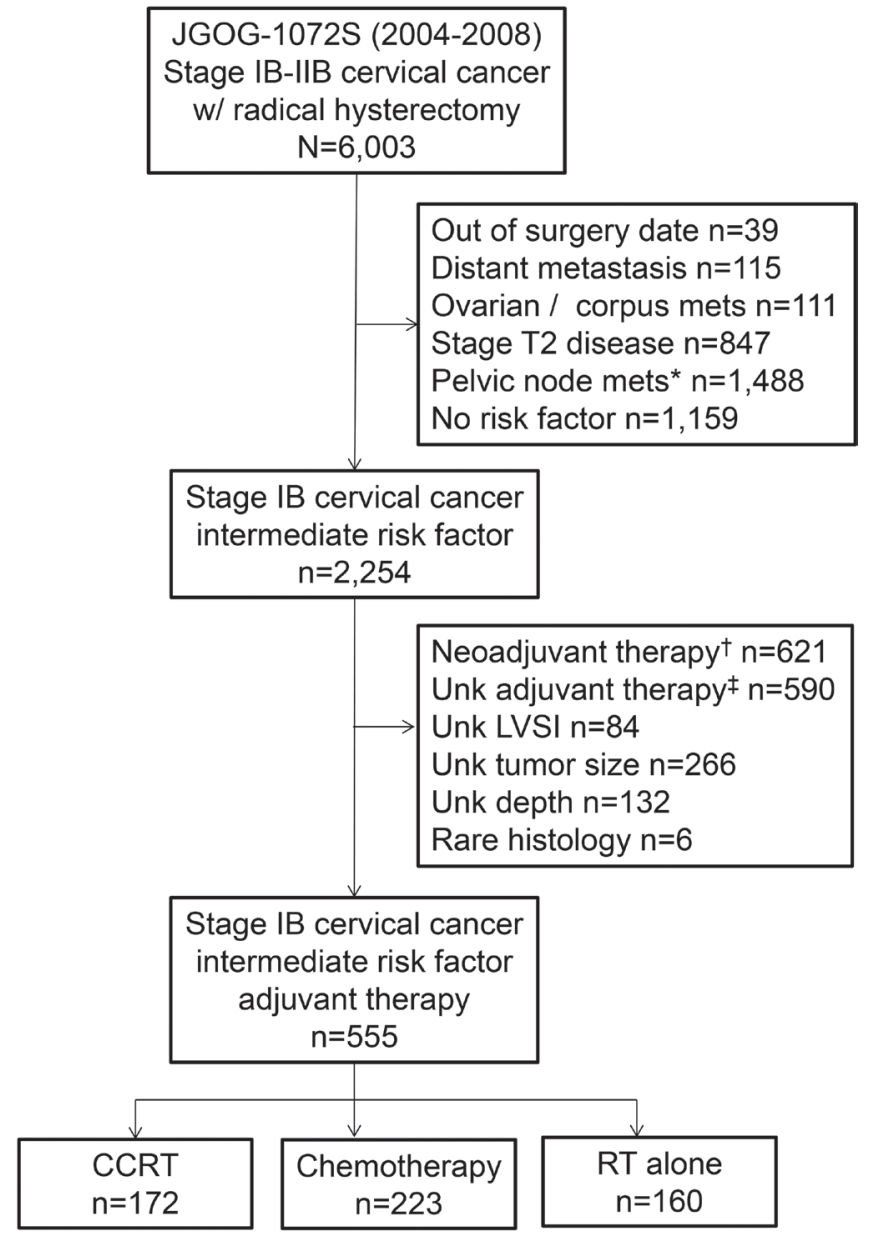

Figure 1: Study selection schema. *Including unknown lymph node status. 'Including unknown neoadjuvant therapy status. ${ }^{*}$ Including no adjuvant therapy status. Abbreviations: JGOG, Japanese Gynecologic Oncology Group; unk, unknown; RT, radiotherapy; and CCRT, concurrent chemo-radiotherapy. 
Table 1: Patient demographics $(N=555)$

\begin{tabular}{|c|c|c|c|c|}
\hline Characteristics & CCRT & Chemotherapy & RT alone & $P$-value \\
\hline No. & $n=172$ & $n=223$ & $n=160$ & \\
\hline Year & & & & 0.36 \\
\hline 2004 & $27(15.7 \%)$ & $30(13.5 \%)$ & $33(20.6 \%)$ & \\
\hline 2005 & $26(15.1 \%)$ & $44(19.7 \%)$ & $28(17.5 \%)$ & \\
\hline 2006 & $39(22.7 \%)$ & $40(17.9 \%)$ & $28(17.5 \%)$ & \\
\hline 2007 & $48(27.9 \%)$ & $57(25.6 \%)$ & $33(20.6 \%)$ & \\
\hline 2008 & $32(18.6 \%)$ & $52(23.3 \%)$ & $38(23.8 \%)$ & \\
\hline Age (mean \pm SD) & $46.0( \pm 10.9)$ & $45.3( \pm 11.0)$ & $47.5( \pm 12.8)$ & 0.18 \\
\hline Stage & & & & 0.32 \\
\hline IB1 & $130(75.6 \%)$ & $181(81.2 \%)$ & $130(81.3 \%)$ & \\
\hline IB2 & $42(24.4 \%)$ & $42(18.8 \%)$ & $30(18.8 \%)$ & \\
\hline Histology & & & & $<0.001$ \\
\hline Squamous & $130(75.6 \%)$ & $105(47.1 \%)$ & $119(74.4 \%)$ & \\
\hline Adenocarcinoma & $33(19.2 \%)$ & $79(35.4 \%)$ & $25(15.6 \%)$ & \\
\hline Adenosquamous & $9(5.2 \%)$ & $39(17.5 \%)$ & $16(10.0 \%)$ & \\
\hline Deep stromal invasion & & & & 0.02 \\
\hline No & $439(22.7 \%)$ & $879(35.4 \%)$ & $45(28.1 \%)$ & \\
\hline Yes & $133(77.3 \%)$ & $144(64.6 \%)$ & $115(71.9 \%)$ & \\
\hline Tumor size & & & & 0.13 \\
\hline$\leq 4.0 \mathrm{~cm}$ & $125(72.7 \%)$ & $181(81.2 \%)$ & $124(77.5 \%)$ & \\
\hline$>4.0 \mathrm{~cm}$ & $47(27.3 \%)$ & $42(18.8 \%)$ & $36(22.5 \%)$ & \\
\hline LVSI & & & & 0.75 \\
\hline Not present & $37(21.5 \%)$ & $55(24.7 \%)$ & $36(22.5 \%)$ & \\
\hline Present & $135(78.5 \%)$ & $1768(75.3 \%)$ & $124(77.5 \%)$ & \\
\hline \multicolumn{5}{|l|}{ Sampled lymph nodes } \\
\hline Pelvic (median IQR) & $26(16)$ & $31(20)$ & $30(18)$ & 0.001 \\
\hline Para-aortic (median IQR)* & $7(8)$ & $8(9)$ & $5(7)$ & 0.045 \\
\hline Risk factor patterns & & & & 0.09 \\
\hline Deep stroma alone & $25(14.5 \%)$ & $35(15.7 \%)$ & $25(15.6 \%)$ & \\
\hline Size alone & $2(1.2 \%)$ & $7(3.1 \%)$ & $4(2.5 \%)$ & \\
\hline LVSI alone & $28(16.3 \%)$ & $67(30.0 \%)$ & $36(22.5 \%)$ & \\
\hline Deep stroma + size & $10(5.8 \%)$ & $13(5.8 \%)$ & $7(4.4 \%)$ & \\
\hline LVSI + deep stroma & $72(41.9 \%)$ & $79(35.4 \%)$ & $63(39.4 \%)$ & \\
\hline LVSI+ size & $9(5.2 \%)$ & $5(2.2 \%)$ & $5(3.1 \%)$ & \\
\hline All 3 factors & $26(15.1 \%)$ & $17(7.6 \%)$ & $20(12.5 \%)$ & \\
\hline
\end{tabular}

Mean ( \pm SD), median (interquartile range), or number (\%) per column are shown. One-way ANOVA test, chi-square test, or Kruskal-Wallis $\mathrm{H}$ test for $P$-values. "Performed in 15 cases in CCRT group, 34 cases in chemotherapy group, and 30 cases for RT group, respectively. Abbreviations: IQR, interquartile range; CCRT, concurrent chemoradiotherapy; and LVSI, lymphovascular space invasion.

statistically similar across the three groups: CCRT group $20 \%$, chemotherapy alone group $30.8 \%$, and $25 \%$ for RT alone group $(P=0.77)$.

There were 326 cases in which the tumor exhibited at least two intermediate-risk factors (chemotherapy alone $n=114$, CCRT $n=117$, and RT alone $n=95$ ). In this sub-group the results were similar to the whole cohort, and adjuvant therapy was not associated with diseasefree survival (5-year rates, $86.9 \%$ for chemotherapy group, $90.2 \%$ for CCRT group, and $85.9 \%$ for RT group, 
Table 2: Adjusting models for disease-free survival and adjuvant therapy $(N=555)$

\begin{tabular}{|c|c|c|c|c|c|c|c|}
\hline \multirow[b]{2}{*}{ Characteristics } & \multirow[b]{2}{*}{ No. } & \multicolumn{2}{|l|}{ Age } & \multicolumn{2}{|c|}{ Age, histology } & \multicolumn{2}{|c|}{ Age, histology, risk factors } \\
\hline & & HR (95\% CI) & $P$-value & HR $(95 \%$ CI $)$ & $P$-value & HR $(95 \%$ CI) & $P$-value \\
\hline \multicolumn{8}{|l|}{ Adjuvant type } \\
\hline CCRT & 172 & 1 & & 1 & & 1 & \\
\hline Chemotherapy alone & 223 & $1.08(0.59-1.98)$ & 0.81 & $0.89(0.48-1.66)$ & 0.71 & $0.98(0.52-1.84)$ & 0.95 \\
\hline RT alone & 160 & $0.95(0.48-1.86)$ & 0.88 & $0.93(0.47-1.83)$ & 0.83 & $0.98(0.50-1.93)$ & 0.95 \\
\hline \multicolumn{8}{|l|}{ Age } \\
\hline$<50$ years & 342 & 1 & & 1 & & 1 & \\
\hline$\geq 50$ years & 213 & $0.75(0.43-1.29)$ & 0.329 & $0.76(0.44-1.31)$ & 0.32 & $0.75(0.44-1.30)$ & 0.31 \\
\hline \multicolumn{8}{|l|}{ Histology } \\
\hline SCC & 354 & & & 1 & & 1 & \\
\hline Non-SCC & 201 & & & $1.91(1.12-3.26)$ & 0.017 & $2.06(1.21-3.51)$ & 0.008 \\
\hline \multicolumn{8}{|l|}{ Deep stromal invasion } \\
\hline No & 163 & & & & & 1 & \\
\hline Yes & 392 & & & & & $1.31(0.74-2.33)$ & 0.36 \\
\hline \multicolumn{8}{|l|}{ Tumor size } \\
\hline$\leq 4.0 \mathrm{~cm}$ & 430 & & & & & 1 & \\
\hline$>4.0 \mathrm{~cm}$ & 125 & & & & & $1.20(0.64-2.25)$ & 0.56 \\
\hline \multicolumn{8}{|l|}{ LVSI } \\
\hline Not present & 128 & & & & & 1 & \\
\hline Present & 427 & & & & & $3.91(1.54-9.95)$ & 0.004 \\
\hline
\end{tabular}

An association of adjuvant treatment type and survival outcome was adjusted by survival factors in Cox proportional-hazards regression models. Three models were tested as above. Significant $P$-values were emboldened. Abbreviations: HR, hazard ratio; CI, confidence interval; CCRT, concurrent chemo-radiotherapy; RT, radiotherapy; SCC, squamous cell carcinoma; and LVSI, lympho-vascular space invasion.
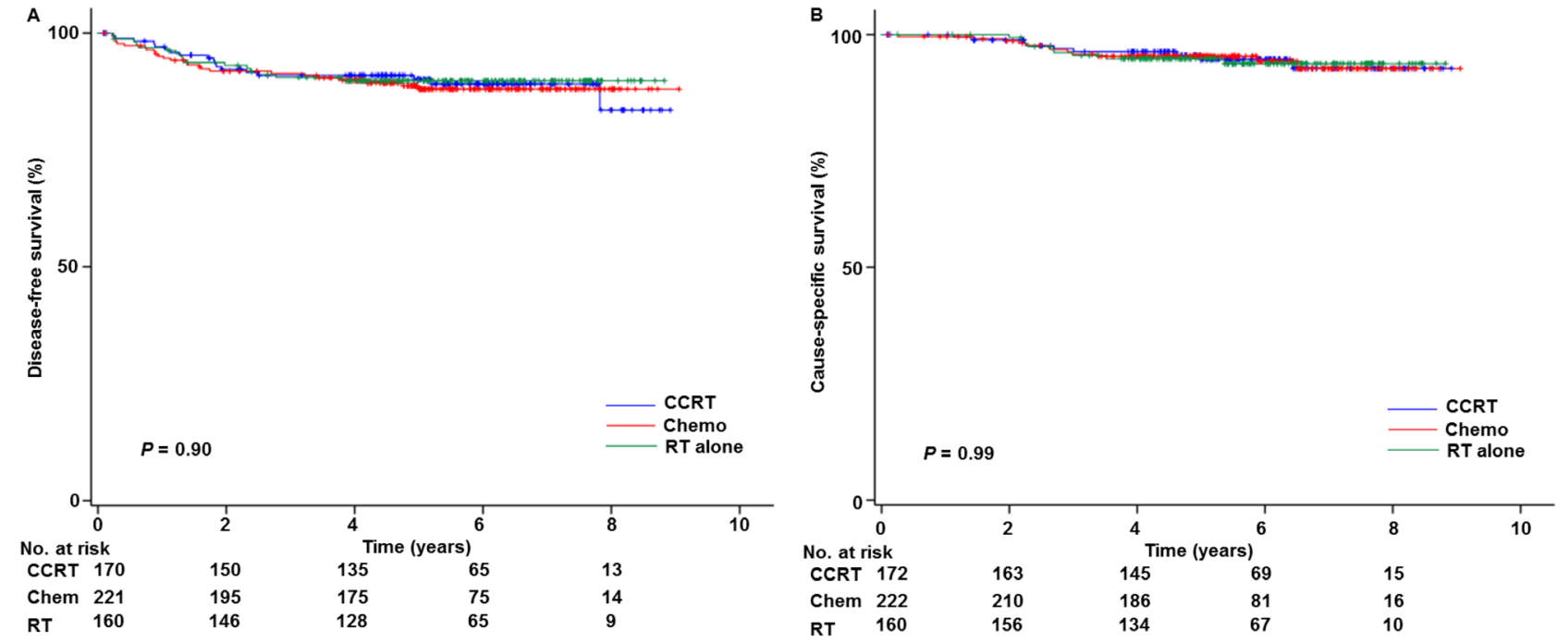

Figure 2: Survival curves based on adjuvant treatment types. Log-rank test for adjusted $P$-values. Survival curves based on adjuvant therapy types are shown for: (A) disease-free survival and (B) cause-specific survival. Abbreviations: CCRT, concurrent chemoradiotherapy; and RT, whole pelvic radiotherapy alone. 
$P=0.86$ ) and cause-specific survival (5-year rates, 94.5\% for chemotherapy group, $93.4 \%$ for CCRT group, and $92.2 \%$ for RT group, $P=0.84$ ). Among 229 cases in which the tumor had only one single intermediate-risk factor, LVSI alone had the lowest disease-free survival compared to other factors although it did not reach statistical significance (5year rate, $87.8 \%$ for LVSI alone, $94.8 \%$ for deep stromal invasion alone, and $100 \%$ for large tumor alone, $P=0.11$ ).

Patterns of recurrence were examined, and local recurrence was seen in 29 cases including 6 cases of vaginal cuff recurrence, while distant recurrence was seen in 33 cases. Adjuvant treatment type was not associated with local recurrence (5-year rates, 5.4\% for chemotherapy group, $4.4 \%$ for CCRT group, and $6.4 \%$ for RT group, $P=0.79$; Figure 3A) and distant recurrence (5-year rates, $5.9 \%$ for chemotherapy group, $5.4 \%$ for CCRT group, and $5.1 \%$ for RT group, $P=0.93$; Figure $3 \mathrm{~B}$ ).

Results of propensity score matching are shown in Supplementary Table 3 . There were no difference in clinicopathological factors between the CCRT group and the chemotherapy group (all, $P>0.05$ ). Women who received postoperative chemotherapy had disease-free survival (5-year rates, $90.4 \%$ versus $89.6 \%$, HR $0.75,95 \%$ CI 0.32 to 1.79 , $P=0.52)$ and cause-specific survival (90.4\% versus $89.6 \%$, HR $0.79,95 \%$ CI 0.21 to $2.92, P=0.72$ ) similar to those who received $\mathrm{CCRT}$.

\section{DISCUSSION}

The key finding of the study is that systemic chemotherapy use was associated with comparable survival compared to radiotherapy-based treatment in women with stage IB cervical cancer and at least one intermediate-risk factor and without a high risk factor. Moreover, our study found that women who had a single intermediate-risk factor with LVSI alone had a survival outcomes similar to those who had multiple intermediate-risk factors.

The effectiveness of systemic chemotherapy as an adjuvant therapy in early-stage cervical cancer in the intermediate-risk group has not been completely studied, and definitive treatment recommendations are currently lacking. Our study findings are similar to prior studies that demonstrated comparable survival outcomes between systemic chemotherapy and CCRT as adjuvant therapy [9, 11]. Because these prior studies were small in sample size (38-47 cases for chemotherapy), our study is more definitive to clarify this association (223 cases for chemotherapy). Therefore, we can respectfully state that postoperative chemotherapy may be as effective as radiation-based therapy.

To date, "classic" criteria of the intermediate-risk group in cervical cancer per the NCCN guidelines require at least two out of the three factors (deep cervical stromal invasion, large tumor, and LVSI) [6]. However, other societies suggest different criteria [13], and the Japan Society of Gynecologic Oncology (JSGO) the intermediaterisk group if any one of the three factors is present in the tumor [7]. We therefore followed the JSGO criteria and included the cases of stage IB cervical cancer with any risk factors as above. However, when we adopted the National Comprehensive Cancer Network (NCCN) guidelines and analyzed a subgroup of cases with multiple risk factors, similar results were demonstrated and chemotherapy use had similar survival outcomes compared to radiotherapy.

Of interest, women whose tumor exhibited only LVSI had survival outcomes similar to those with multiple risk factors (5-year disease-free survival rate for LVSI alone versus multiple risk factors, $87.9 \%$ versus 85.9-90.2\%). Recurrence risks were minimal when tumors exhibited only large size or deep cervical stromal invasion (5-year disease-free survival $94.8 \%$ and 100\%,
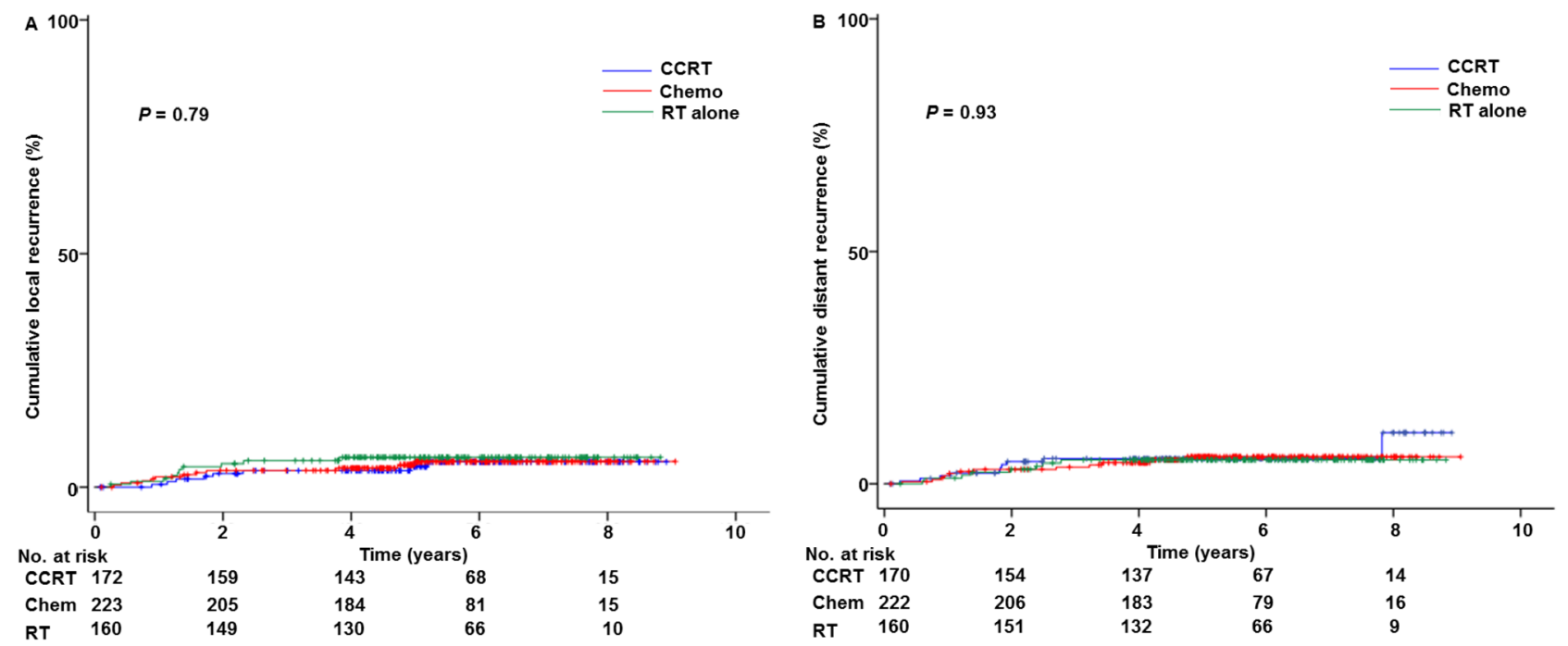

Figure 3: Cumulative incidence curves based on adjuvant treatment types. Log-rank test for adjusted $P$-values. Cumulative incidence curves based on adjuvant therapy types are shown for: (A) loco-regional recurrence and (B) distant recurrence. Abbreviations: CCRT, concurrent chemo-radiotherapy; and RT, whole pelvic radiotherapy alone. 
Table 3: Adjusting models for cause-specific survival and adjuvant therapy $(N=555)$

\begin{tabular}{|c|c|c|c|c|c|c|c|}
\hline \multirow[b]{2}{*}{ Characteristics } & \multirow[b]{2}{*}{ No. } & \multicolumn{2}{|l|}{ Age } & \multicolumn{2}{|c|}{ Age, histology } & \multicolumn{2}{|c|}{ Age, histology, risk factors } \\
\hline & & HR $(95 \%$ CI $)$ & $P$-value & HR $(95 \%$ CI $)$ & $P$-value & HR $(95 \%$ CI) & $P$-value \\
\hline \multicolumn{8}{|l|}{ Adjuvant type } \\
\hline CCRT & 172 & 1 & & 1 & & 1 & \\
\hline Chemotherapy alone & 223 & $1.03(0.43-2.44)$ & 0.95 & $0.85(0.34-2.08)$ & 0.71 & $1.02(0.41-2.54)$ & 0.96 \\
\hline RT alone & 160 & $1.05(0.42-2.64)$ & 0.92 & $1.03(0.41-2.59)$ & 0.95 & $1.13(0.44-2.85)$ & 0.80 \\
\hline \multicolumn{8}{|l|}{ Age } \\
\hline$<50$ years & 342 & 1 & & 1 & & 1 & \\
\hline$\geq 50$ years & 213 & $0.77(0.36-1.65)$ & 0.50 & $0.79(0.37-1.68)$ & 0.54 & $0.81(0.38-1.73)$ & 0.659 \\
\hline \multicolumn{8}{|l|}{ Histology } \\
\hline SCC & 354 & & & 1 & & 1 & \\
\hline Non-SCC & 201 & & & $1.85(0.87-3.892)$ & 0.11 & $2.17(1.02-4.62)$ & 0.045 \\
\hline \multicolumn{8}{|l|}{ Deep stromal invasion } \\
\hline No & 163 & & & & & 1 & \\
\hline Yes & 392 & & & & & $1.02(0.47-2.21)$ & 0.97 \\
\hline \multicolumn{8}{|l|}{ Tumor size } \\
\hline$\leq 4.0 \mathrm{~cm}$ & 430 & & & & & 1 & \\
\hline$>4.0 \mathrm{~cm}$ & 125 & & & & & $2.879(1.29-6.00)$ & 0.009 \\
\hline \multicolumn{8}{|l|}{ LVSI } \\
\hline Not present & 128 & & & & & 1 & \\
\hline Present & 427 & & & & & $11.4(1.52-84.8)$ & 0.018 \\
\hline
\end{tabular}

An association of adjuvant treatment type and survival outcome was adjusted by survival factors in Cox proportional-hazards regression models. Three models were tested as above. Abbreviations: HR, hazard ratio; CI, confidence interval; CCRT, concurrent chemo-radiotherapy; RT, radiotherapy; SCC, squamous cell carcinoma; and LVSI, lympho-vascular space invasion.

respectively). Therefore, LVSI alone may need to be considered as an intermediate-risk factor. Further study to validate and prove our results is warranted.

In a sensitivity analysis, we examined survival outcome of women who received CCRT compared to RT alone. The current guidelines for the intermediaterisk group recommend whole pelvic radiotherapy alone without concurrent administration of chemotherapy, and the use of CCRT is optional $[6,7]$. In review of the literature, there were multiple cohort studies examined the effectiveness of CCRT over RT alone for the intermediaterisk group [14, 15], suggesting improved survival with CCRT use. Conversely, other investigators concluded that CCRT does not improve survival when compared to radiotherapy alone in the intermediate-risk group [16]. Our study also did not demonstrate a difference in survival between CCRT and RT alone groups. Currently, there is an ongoing phase III randomized controlled trial comparing CCRT versus RT alone in women with surgically treated intermediate-risk early-stage cervical cancer (GOG-263) [17]. The estimated time for the completion of accrual is December 2020, and this trial will ultimately answer the utility of CCRT for this patient population.
The benefit of offering systemic chemotherapy over radiotherapy is to minimize complications from pelvic irradiation administered after radical pelvic surgery. This rationale was based on a general consensus that demonstrated high complication rates with pelvic irradiation after radical hysterectomy compared to surgery alone [18]. In the intermediate-risk group, one prior retrospective study reported a higher adverse event rate in the CCRT group compared to the chemotherapy group [11]. Our study did not have information to assess postoperative complications. To date, there is no headto-head trial directly comparing efficacy and adverse events between adjuvant chemotherapy and radiotherapy in intermediate-risk early-stage cervical cancer. In Japan, JGOG is going to launch a phase III trial comparing adjuvant chemotherapy and CCRT in women with highrisk stage IB-IIB cervical cancer (AFTER trial). Although the patient population is not an intermediate-risk group, this trial will answer the question regarding the adverse event related to adjuvant therapy.

Strengths of the study included a sample size that is one of the largest in the literature. In addition, the dataset is considerably clear with our rigorous inclusion and exclusion criteria, making interpretation of the results 
more reliable. However, there are multiple limitations in this study. First, this is a retrospective study that may miss confounding factors in the analysis. For example, we do not know the treatment allocation for chemotherapy versus other treatment types. Indeed, there are various practice patterns for adjuvant therapy in intermediaterisk early-stage cervical cancer in Japan [19], and this can be a major drawback of the study resulting in selection bias. Second, there are multiple chemotherapy regimens and administered cycles used in the chemotherapy group (heterogeneity), and we were not able to recommend any specific chemotherapy choice over others.

Weaknesses of the study included that our study population had a fairly favorable survival outcome with the limited number of survival events despite the adequate median follow-up of more than five years. For this reason, an adjustment model with five covariates for cause-specific survival may result in over-adjustment given the number of cervical cancer mortality events. However, two other adjustment models for cause-specific survival were consistent to support the current findings, and this association of chemotherapy and cause-specific survival is likely true. While central pathology review was not performed to confirm that intermediate-risk criteria was met, these surgical-pathological factors are well-established histopathological findings and thus inter-observer-variability is less likely to differ across the pathologists.

The intermediate-risk criteria for deep stromal invasion was recorded as outer versus inner half and tumor size was recorded for $>4$ versus $\leq 4 \mathrm{~cm}$ in this surgical database per the JSGO criteria, and thus, we were not able to apply the Gynecologic Oncology Group (GOG) or Korean criteria for the intermediate-risk in cases where the depth of invasion was defined as outer third $[4,20]$. In this study, we used the JSGO criteria because this study was conducted in the society designated institutions and the validation of the society criteria has not been tested in largescale study population. However, when we examined the cases with any two or more risk factors, survival outcome of our study population seems comparable to that seen in the GOG-92 trial (2-year disease-free survival rate 88\%) [4].

In summary, our study suggests that systemic chemotherapy can be an alternative effective treatment choice as adjuvant therapy for women with intermediaterisk stage IB cervical cancer. This option can be particularly suitable in patients who are not the best candidates for radiotherapy such as those with pelvic adhesive disease, intraoperative abdomino-pelvic injury, radiation intolerance, and non-compliance for the radiation treatment schedule. Moreover, because increased chemotherapy cycles did not improve survival, and women in the intermediate-risk group have a generally good prognosis, the utility of a reduction in the number of chemotherapy cycles may be considered to minimize the toxicity of chemotherapy. Finally, the current intermediate- risk criteria per the JSGO guidelines need to be revised, as the present criteria may result in overtreatment in cases where the prognosis is generally favorable. Based on our results, at least two risk factors as well as LVSI alone may meet criteria for stratification into the intermediate-risk category. Establishing stricter criteria weighing treatment benefits and risks are warranted.

\section{MATERIALS AND METHODS}

\section{Eligibility}

This was a retrospective analysis of the previously organized nation-wide large-scale observational study conducted in 116 Japanese Gynecologic Oncology Group (JGOG) designated institutions [20-22]. We collected consecutive cases of women with stage IB-IIB cervical cancer who underwent a radical hysterectomy between January 1, 2004 and December 31, 2008. The study period for the data acquisition was between October 1, 2012 and February 28, 2013. Institutional Review Board approval was obtained at Tottori University, which served as the host institution, and JGOG-participating institutions reviewed the protocol and obtained Institutional Review Board approval as indicated.

Eligibility criteria for this study were women with stage IB cervical cancer who met criteria for intermediaterisk disease, and received adjuvant therapy following type III radical hysterectomy and pelvic lymphadenectomy. Per the JSGO treatment guidelines for cervical cancer, the intermediate-risk group is defined as cervical cancer in which the tumor is confined to the cervix without parametrial or lymph node involvement, and exhibits any one of the following three factors: large cervical tumor $>4 \mathrm{~cm}$, deep cervical stromal invasion (outer half), and LVSI [7]. We limited the histology types only to squamous cell carcinoma, adenocarcinoma, and adenosquamous for comparison with a prior study [4]. Women were excluded from the study if high-risk criteria were present (pelvic lymph node metastasis, parametrial tumor involvement, and surgical margin tumor involvement) or if no surgicalpathological risk factor was identified. We also excluded women with tumor involving in para-aortic lymph nodes, ovaries, or uterine corpus. Women who received neoadjuvant therapy, unknown adjuvant therapy, or received a battery of both systemic chemotherapy and radiotherapy were also excluded.

\section{Clinical information}

Clinical and tumor information abstracted from medical and pathological records included age, histologic subtype, clinical and pathological stages, tumor size, pelvic and para-aortic lymph node status (including the number sampled), parametrial involvement, deep stromal invasion, LVSI, uterine corpus involvement, ovarian 
involvement, and the presence of distant metastasis. Adjuvant treatment information included the following three modalities: concurrent chemo-radiotherapy with pelvic irradiation and weekly chemotherapy (CCRT group), systemic chemotherapy alone (chemotherapy group), and whole pelvic radiotherapy alone (RT group). Among women who received chemotherapy, the type of chemotherapy, the number of administered cycles, and toxicity were recorded.

Survival information included disease-free and cause-specific survival. Disease-free survival was defined as the time interval between the hysterectomy and the first recurrence. Cause-specific survival was defined as the time interval between the hysterectomy date and death due to cervical cancer. The patients were censored if patients were alive at the last follow-up or had died due to another cause. Among women who developed recurrent disease, locations of recurrence were grouped into local recurrence (vaginal cuff and/or pelvis) and distant recurrence (any site other than local).

\section{Statistical analysis}

The primary interest of this analysis was to examine survival of women with intermediate-risk stage IB cervical cancer who received systemic chemotherapy compared to those who received CCRT. The secondary interest of analysis was to assess recurrence patterns based on adjuvant therapy types. A sensitivity analysis was performed to examine cases with at least two intermediaterisk factors by adopting the NCCN guidelines [6]. In addition, another sensitivity analysis was performed to compare survival of the CCRT group to the RT alone group per an ongoing phase III clinical trial examining intermediate-risk cervical cancer (GOG-263) [17]. Similarly, a sensitivity analysis was performed to examine survival of the systemic chemotherapy group compared to the RT alone group as radiotherapy alone is the current standard adjuvant therapy for patients in the intermediaterisk group [4].

The statistical significance of continuous variables among multiple groups was assessed by either the oneway ANOVA test or by the Kruskal-Wallis $\mathrm{H}$ test as appropriate. Statistical significance of categorical and ordinal variables was assessed by the chi-square test. The Kaplan-Meier method was used to construct survival curves and cumulative risk curves [23], and statistical significance between these curves was determined by the log-rank test. Cox proportional hazard regression models were used to assess the independent association of adjuvant therapy and survival by adjusting for a priori survival factors [24]. In this study, we tested three different models. The first model was adjusted for age alone ( $<50$ versus $\geq 50$ years), the second model was adjusted for age and histology type (squamous versus non-squamous), and the third model was adjusted for age, histology, and histo-pathological factors (tumor size, depth of myometrial tumor invasion, and LVSI). The magnitude of statistical significance was expressed with an adjusted-HR and 95\% CI.

We also performed a propensity score matching to adjust the background differences between the chemotherapy group and the CCRT group. Propensity score for chemotherapy use was computed for each case determined by multivariable logistic regression analysis. In the model, year of diagnosis, age, stage, histology types, extent of lymphadenectomy and tumor factors (deep stromal invasion, tumor size, and LVSI). An automated algorithm was used for one-to-one matching between the two groups (cutoff, 1\%).

All statistical analyses were based on two-side hypothesis, and a $P$-value of less than 0.05 was considered significant. Statistical Package for Social Sciences (IBM SPSS, version 24.0, Armonk, NY, USA) was used for the analysis. The STROBE guidelines for a retrospective observational study were consulted to outline this study [25].

\section{ACKNOWLEDGMENTS}

We thank all the JGOG institutions participated in this study and the JGOG Cervical Cancer Committee members for administrative work for the study. We also thank Dr. Brendan H. Grubbs, MD, for his scientific input to the study.

\section{CONFLICTS OF INTEREST}

None declared.

\section{REFERENCES}

1. Torre LA, Bray F, Siegel RL, Ferlay J, Lortet-Tieulent J, Jemal A. Global cancer statistics, 2012. CA Cancer J Clin. 2015; 65:87-108.

2. Waggoner SE. Cervical cancer. Lancet. 2003; 361:2217-25.

3. Delgado G, Bundy B, Zaino R, Sevin BU, Creasman WT, Major F. Prospective surgical-pathological study of disease-free interval in patients with stage IB squamous cell carcinoma of the cervix: a Gynecologic Oncology Group study. Gynecol Oncol. 1990; 38:352-7.

4. Sedlis A, Bundy BN, Rotman MZ, Lentz SS, Muderspach LI, Zaino RJ. A randomized trial of pelvic radiation therapy versus no further therapy in selected patients with stage IB carcinoma of the cervix after radical hysterectomy and pelvic lymphadenectomy: A Gynecologic Oncology Group Study. Gynecol Oncol. 1999; 73:177-83.

5. Peters WA 3rd, Liu PY, Barrett RJ 2nd, Stock RJ, Monk BJ, Berek JS, Souhami L, Grigsby P, Gordon W Jr, Alberts DS. Concurrent chemotherapy and pelvic radiation therapy compared with pelvic radiation therapy alone as adjuvant therapy after radical surgery in high-risk early-stage cancer of the cervix. J Clin Oncol. 2000; 18:1606-13. 
6. Cervical cancer. NCCN Clinical Practice Guidelines in Oncology (NCCN Guidelines) <http://www.ncen.org> (accessed 7/1/2017).

7. Ebina Y, Yaegashi N, Katabuchi H, Nagase S, Udagawa Y, Hachisuga T, Saito T, Mikami M, Aoki Y, Yoshikawa H. Japan Society of Gynecologic Oncology guidelines 2011 for the treatment of uterine cervical cancer. Int J Clin Oncol. 2015; 20:240-8.

8. Rotman M, Sedlis A, Piedmonte MR, Bundy B, Lentz SS, Muderspach LI, Zaino RJ. A phase III randomized trial of postoperative pelvic irradiation in Stage IB cervical carcinoma with poor prognostic features: follow-up of a gynecologic oncology group study. Int J Radiat Oncol Biol Phys. 2006; 65:169-76.

9. Lee KB, Lee JM, Ki KD, Lee SK, Park CY, Ha SY. Comparison of adjuvant chemotherapy and radiation in patients with intermediate risk factors after radical surgery in FIGO stage IB-IIA cervical cancer. Int J Gynecol Cancer. 2008; 18:1027-31.

10. Lee TY, Jeung YJ, Lee CJ, Kim HY, Kim SH, Kim WG. Promising treatment results of adjuvant chemotherapy following radical hysterectomy for intermediate risk stage 1B cervical cancer. Obstet Gynecol Sci. 2013; 56:15-21.

11. Yu H, Zhang L, Du X, Sheng X. Postoperative adjuvant chemotherapy combined with intracavitary brachytherapy in early-stage cervical cancer patients with intermediate risk factors. Onco Targets Ther. 2016; 9:7331-7335.

12. Takeshima N, Umayahara K, Fujiwara K, Hirai Y, Takizawa K, Hasumi K. Treatment results of adjuvant chemotherapy after radical hysterectomy for intermediate- and highrisk stage IB-IIA cervical cancer. Gynecol Oncol. 2006; 103:618-22.

13. Ryu SY, Kim MH, Nam BH, Lee TS, Song ES, Park CY, Kim JW, Kim YB, Ryu HS, Park SY, Kim KT, Cho CH, Lee $\mathrm{C}$, et al. Intermediate-risk grouping of cervical cancer patients treated with radical hysterectomy: a Korean Gynecologic Oncology Group study. Br J Cancer. 2014; 110:278-85.

14. Song S, Song C, Kim HJ, Wu HG, Kim JH, Park NH, Song YS, Kim JW, Kang SB, Ha SW. 20 year experience of postoperative radiotherapy in IB-IIA cervical cancer patients with intermediate risk factors: impact of treatment period and concurrent chemotherapy. Gynecol Oncol. 2011; 124:63-7.

15. Okazawa M, Mabuchi S, Isohashi F, Suzuki O, Yoshioka Y, Sasano T, Ohta Y, Kamiura S, Ogawa K, Kimura T.
Impact of the addition of concurrent chemotherapy to pelvic radiotherapy in surgically treated stage IB1-IIB cervical cancer patients with intermediate-risk or high-risk factors: a 13-year experience. Int J Gynecol Cancer. 2013; 23:567-75.

16. Qin AQ, Liang ZG, Ye JX, Li J, Wang JL, Chen CX, Song HL. Significant Efficacy of Additional Concurrent Chemotherapy with Radiotherapy for Postoperative Cervical Cancer with Risk Factors: a Systematic Review and Meta-analysis. Asian Pac J Cancer Prev. 2016; 17:3945-51.

17. https://clinicaltrials.gov/ct2/show/NCT01101451 (accessed 7/1/2017).

18. Landoni F, Maneo A, Colombo A, Placa F, Milani R, Perego P, Favini G, Ferri L, Mangioni C. Randomised study of radical surgery versus radiotherapy for stage Ib-IIa cervical cancer. Lancet. 1997; 350:535-40.

19. Ikeda Y, Furusawa A, Kitagawa R, Tokinaga A, Ito F, Ukita M, Nomura H, Yamagami W, Tanabe H, Mikami M, Takeshima N, Yaegashi N. Practice patterns of adjuvant therapy for intermediate/high recurrence risk cervical cancer patients in Japan. J Gynecol Oncol. 2016; 27:e29

20. Matsuo K, Shimada M, Aoki Y, Sakamoto M, Takeshima N, Fujiwara H, Matsumoto T, Mikami M, Sugiyama T. Comparison of adjuvant therapy for node-positive clinical stage IB-IIB cervical cancer: Systemic chemotherapy versus pelvic irradiation. Int J Cancer. 2017; 141:1042-51.

21. Matsuo K, Shimada M, Yamaguchi S, Kanao H, Nakanishi T, Saito T, Kamiura S, Iwata T, Mikami M, Sugiyama T. Identifying a candidate population for ovarian conservation in young women with clinical stage IB-IIB cervical cancer. Int J Cancer. 2017 Oct 4. https://doi.org/10.1002/ijc.31084. [Epub ahead of print].

22. Matsuo K, Shimada M, Mikami M. Ovarian conservation for young women with clinical stage IB-IIB cervical cancer in Japan. J Gynecol Oncol. 2017; 28:e60.

23. Kaplan EL, Meier P. Nonparametric Estimation from Incomplete Observations. J Am Stat Assoc. 1958; 53:457-81.

24. Cox DR. Regression Models and Life-Tables. J R Stat Soc Series B Stat Methodol. 1972; 34:187-220.

25. von Elm E, Altman DG, Egger M, Pocock SJ, Gøtzsche PC, Vandenbroucke JP, and STROBE Initiative. Strengthening the Reporting of Observational Studies in Epidemiology (STROBE) statement: guidelines for reporting observational studies. BMJ. 2007; 335: 806-8. 